\title{
Cervical screening rates for women vaccinated against human papillomavirus
}

\section{Alison C Budd \\ SeniorData}

Julia M L Brotherton BMed(Hons), MPH(Hons), FAFPHM

Medical Director Nation HPV Vaccination Program Register, ${ }^{2}$ and Honorary Senior Fellow

Dorota M Gertig MBBS(Hons), ScD, FAFPHM Medical Director, Victorian Cervical Cytology Registry, and Honorary Principal Fellow ${ }^{3}$

Theresa Chav BPsychS Data Analyst

Kelly T Drennan BHIthSc, PGCertCommDe Research Fellow

Marion Saville MBChB, FIAC GradDipMed(ClinEpi) Executive Director ${ }^{2}$

1 Cancer and Screening Unit, Australian Institut of Health and Welfare, Canberra, ACT.

2 Victorian Cytology Service Melbourne, VIC.

3 Centre for Epidemiology and Biostatistics, Melbourne School of Population and Global Health, University of Melbourne Melbourne, VIC

msaville@vcs.org.au

MJA 2014; 201: 279-282 doi: 10.5694/mial4.0002 ustralia has a well established cervical screening program that currently recommends 2-yearly Pap tests for women aged 18 years (or 2 years after commencement of sexual activity, whichever is later) to 69 years. The program is supported by opt-off cervical cytology registers ("Pap test registers") in each state and territory that record the results of cervical cytology tests, cervical histopathology tests and human papillomavirus (HPV) DNA tests. The relatively high level of participation in Australia's National Cervical Screening Program has led to halving of cervical cancer incidence and mortality rates. ${ }^{1}$

In April 2007, Australia initiated a national, publicly funded HPV vaccination program, using a threedose schedule of a quadrivalent HPV recombinant vaccine that protects against HPV types 16 and 18 (which are responsible for $70 \%-80 \%$ of cervical cancer cases in Australia ${ }^{2}$ ) and types 6 and 11 (which cause genital warts). The program vaccinates 12-year-old and 13-year-old girls in schools and included a catch-up vaccination program for 14-18-yearold girls in schools and 18-26-yearold women in community-based settings until the end of 2009. All communications provided to vaccinated women emphasised the importance of continuing regular cervical screening. This is because the vaccine does not prevent all HPV types that cause cervical cancer and, as a prophylactic vaccine, it will not protect women who were vaccinated under the catch-up program but had already acquired HPV through sexual activity.

Analysis of participation trends from 2004-2005 to 2010-2011 in Victoria for women aged 20-24 and 25-29 years shows that, after a small peak in 2006-2007, coinciding with the start of the National HPV Vaccination Program, participation in cervical screening declined steadily (Box 1$){ }^{1}$ For women aged $20-24$ years, participation over the period 2006-2007 to 2010-2011 declined from

\section{Abstrac}

Objective: To compare cervical screening rates for women vaccinated with a quadrivalent human papillomavirus (HPV) vaccine with those for unvaccinated women, to address concerns that vaccinated women may not be participating in cervical screening.

Design, setting and participants: Cross-sectional analysis of linked data from the Victorian Cervical Cytology Registry and the National HPV Vaccination Program Register for 20-29-year-old women in Victoria, Australia, for the period 1 January 2009 to 31 December 2011.

Main outcome measures: Screening participation rates for vaccinated and unvaccinated women.

Results: Participation in cervical screening during the 2-year period 2010-2011 was significantly lower in 20-24-year-old vaccinated women compared with unvaccinated women of the same age (37.6\% v 47.7\%, a 10.1 percentage point difference [95\% Cl, 9.7-10.6]; $P<0.001$ ) and significantly lower in 25-29-yearold vaccinated women compared with unvaccinated women of the same age (45.2\% v 58.7\%, a 13.5 percentage point difference [95\% Cl, 13.1\%-13.9\%]; $P<0.001)$. Similar results were observed for participation during the 3 -year period 2009-2011.

Conclusions: Despite education messages provided to young women, our results suggest that vaccinated women are being screened at lower rates than unvaccinated women in Australia. While some degree of undermatching of women in the study may have occurred, this cannot wholly explain our findings. Effective implementation of Individual Healthcare Identifiers to health records, including registry records, is needed to prevent potential undermatching of individuals in future linkage studies. In the meantime, efforts to increase participation in cervical screening by vaccinated women are needed.

$48.1 \%$ to $41.7 \%$; for women aged $25-29$ years, it declined from $58.9 \%$ to $52.3 \%$. This trend was mirrored in national participation rates over this period. ${ }^{1}$

We aimed to evaluate the effect of HPV vaccination on participation in cervical screening in Victoria to address concerns that vaccinated women may not be continuing to participate in cervical screening, which would put them at risk of developing cervical cancers caused by vaccinetype HPV infections acquired before vaccination and non-vaccine HPV types.

\section{Methods}

We conducted a cross-sectional analysis of linked data from the Victorian Cervical Cytology Registry (VCCR) and the National HPV Vaccination Program Register (NHVPR). Ethics approval for the study was obtained from the Department of Health Human Research Ethics Committee and Australian Institute of Health and Welfare (AIHW) Ethics Committee.

\section{Data collection}

The VCCR captures cervical screening results for all female residents of Victoria (a population of more than 2.7 million), including cervical cytology and cervical histopathology test results obtained directly from laboratories. Fewer than $1 \%$ of women "opt off" the VCCR. ${ }^{3}$ The NHVPR records HPV vaccine doses administered nationwide. Doses administered through schools are received from school immunisation providers, which are predominantly local councils and health departments. ${ }^{4}$ Notification data from these providers are believed to be virtually complete, whereas notification data for women aged 18 years and over who were vaccinated in the catch-up program are believed to underestimate vaccination coverage by about $10 \% .5,6$

\section{Data linkage}

Cross-referencing of vaccination data against data in cervical cytology registers, to monitor the effectiveness of $\mathrm{HPV}$ vaccine, is accommodated by 
1 Two-year participation in cervical screening by Victorian women aged 20-24, 25-29 and 20-69 years, $2004-2005$ to $2010-2011$

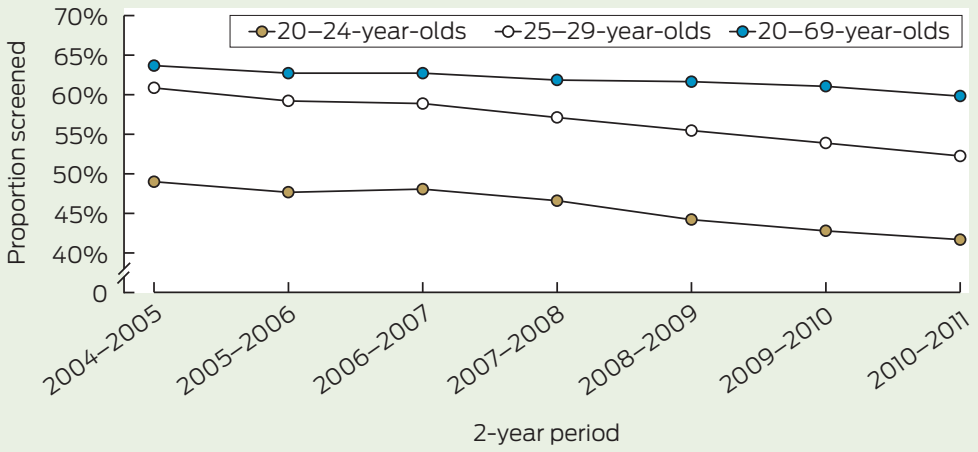

legislation that enables operation of the NHVPR. However, the VCCR has no similar provision, so individual identifiers could not be used for the data linkage in this study.

A deterministic linkage procedure was undertaken to link records from the VCCR (which included Victorian women who are age-eligible for the $\mathrm{HPV}$ vaccine as per product indication [date of birth on or after 1 July 1962]) to records from the NHVPR (which included all Australian women who received the HPV vaccine before 1 January 2012).

Data from the VCCR and NHVPR were de-identified in the same way. A randomly generated record identification number was assigned to women in each register. The Data Linkage Unit of the AIHW generated a series of 22 linkage keys using combinations of variables such as selected letters from given name and surname, perturbed date of birth, postcode, and parts of the Medicare number. The quality of these linkage keys was assessed by calculating the proportion of unique combinations that each would be likely to produce. The first 16 linkage keys produced a set of unique combinations with the best balance between incorrectly accepting a given record pair as the same person versus failing to accept a given record pair as the same person, and these were used to link records.
In a sensitivity analysis, we compared estimates of screening participation rates based on the 16 selected linkage keys with those based on all 22 linkage keys, including the six lowerquality linkage keys.

\section{Participation}

Screening participation rates by vaccination status were estimated for women aged 20-29 years at the time of screening between 1 January 2009 and 31 December 2011, as this group was eligible for both catch-up vaccination and cervical screening. We also explored participation among women aged $30-34$ years by vaccination status; during the study period, almost all vaccinated women in this age group would have been vaccinated electively, rather than given free vaccination as part of the catchup program.

Participation rates were calculated as the proportion of women residing in Victoria who had at least one Pap test over the 2-year period 2010-2011 and the 3-year period 2009-2011, using Australian Bureau of Statistics estimated resident population estimates. The population was not adjusted to account for women who had had a hysterectomy because of the very low rates of hysterectomy in women younger than 30 years. Vaccination status was assigned to screened women according to their status at the beginning of the period of interest.

A sensitivity analysis was conducted to assess the impact of $10 \%$ undernotification of vaccination (ie, assuming $10 \%$ more of the population were vaccinated and that $10 \%$ more of the screened women were vaccinated), as indicated by independent survey data on vaccination coverage. ${ }^{6}$

\section{Results}

\section{Screening among 20-29-year-olds}

Using our linked dataset, we found that 2-year participation in cervical screening was significantly lower for vaccinated women than for unvaccinated women (Box 2). The difference for women aged 20-24 years was 10.1 percentage points $(37.6 \% \mathrm{v}$ $47.7 \%$ ) and the difference for women aged 25-29 years was 13.5 percentage points (45.2\% v 58.7\%).

A similar pattern was apparent for 3-year participation (Box 2). The difference for women aged 20-24 years was 11.7 percentage points $(49.7 \% \mathrm{v}$ $61.4 \%$ ) and the difference for women aged 25-29 years was 21.7 percentage points (54.2\% v $75.9 \%)$.

The groups of women aged 20-24 years who received one, two and three doses of the HPV vaccine had similar levels of participation (37.6\%, $40.5 \%$ and $36.8 \%$, respectively; $\chi^{2}$ test, $P<0.001 ; \chi^{2}$ test for trend, $P<0.001$ ). In contrast, there was a positive association between number of vaccine doses received and screening participation for women aged 25-29 years (one dose, 37.9\%; two doses, $42.1 \%$; three doses, $48.1 \%$; $\chi^{2}$ test, $P<0.001$; $\chi^{2}$ test for trend, $\left.P<0.001\right)$. The same pattern held true for 3-year participation (data not shown).

\section{Timing of first Pap tests}

Of women aged 18-26 years who had their first Pap test on or after 1 April 2007, 11.1\% had their first Pap test either on the same day as their first dose

2 Participation in cervical screening by Victorian women, by age and vaccination status, over a 2-year period and a 3-year period

\begin{tabular}{|c|c|c|c|c|c|c|c|c|}
\hline \multirow[b]{2}{*}{ Age } & \multicolumn{4}{|c|}{ 2-year participation (2010-2011) } & \multicolumn{4}{|c|}{ 3-year participation (2009-2011) } \\
\hline & $\begin{array}{l}\text { Vaccinated } \\
\text { women }\end{array}$ & $\begin{array}{l}\text { Unvaccinated } \\
\text { women }\end{array}$ & $\begin{array}{c}\text { Percentage point } \\
\text { difference }(95 \% \mathrm{Cl})\end{array}$ & $P$ & $\begin{array}{l}\text { Vaccinated } \\
\text { women }\end{array}$ & $\begin{array}{l}\text { Unvaccinated } \\
\text { women }\end{array}$ & $\begin{array}{c}\text { Percentage point } \\
\text { difference }(95 \% \mathrm{Cl})\end{array}$ & $P$ \\
\hline $20-24$ years & $37.6 \%$ & $47.7 \%$ & $10.1(9.7-10.6)$ & $<0.001$ & $49.7 \%$ & $61.4 \%$ & $11.7(11.3-12.1)$ & $<0.001$ \\
\hline $25-29$ years & $45.2 \%$ & $58.7 \%$ & 13.5 (13.1-13.9) & $<0.001$ & $54.2 \%$ & $75.9 \%$ & $21.7(21.3-22.1)$ & $<0.001$ \\
\hline
\end{tabular}


of vaccine $(4.1 \%)$ or between their first and last vaccine doses (7.0\%). Among women aged $<18$ years, $10.6 \%$ had their first Pap test either on the same day as their first dose of vaccine $(1.5 \%)$ or between their first and last vaccine doses $(9.1 \%)$.

\section{Screening among 30-34-year-olds}

A small proportion of 30-34-yearold women (ie, those outside the eligible age range) electively received the HPV vaccine: $5 \%$ in $2009,8 \%$ in 2010 and $12 \%$ in 2011. In 2011, women who were aged 26 years in 2007, and thus eligible for funded vaccination, turned 30 years old. In the 30-34-year age group, screening participation rates were significantly and substantially lower for vaccinated women than for unvaccinated women (2-year participation: $27.5 \%$ v $61.3 \%, 33.8$ percentage point difference $[95 \% \mathrm{CI}$, 33.1-34.5], $P<0.001$; 3-year participation: $21.4 \%$ v $77.1 \%$, 55.7 percentage point difference [95\% CI, 54.9-56.5], $P<0.001)$.

\section{Sensitivity analyses}

Results were largely unaltered (participation estimates within 1\%) in the sensitivity analysis that assumed $10 \%$ undernotification of HPV vaccination. In the sensitivity analysis in which we accepted matching records based on lower-quality linkage keys, findings for participation were robust for the 25-29-year age group (2-year participation: $49.4 \%$ vaccinated v 54.2\% unvaccinated; 3-year participation: $59.3 \%$ vaccinated $\mathrm{v}$ $72.0 \%$ unvaccinated) but not for the 20-24-year age group, in which vaccinated women appeared slightly more likely to participate in screening (2year participation: $42.7 \%$ vaccinated v 38.9\% unvaccinated; 3-year participation: $55.8 \%$ vaccinated v $53.6 \%$ unvaccinated).

\section{Discussion}

This study provides the first direct evidence that women vaccinated against HPV in the community catchup program are being screened for cervical cancer at lower rates than unvaccinated women in Australia. (The large Victorian sample we used is considered representative of Australia.) Participation in cervical screening was significantly lower in vaccinated women aged 20-24 and 25-29 years than in unvaccinated women. This persisted when probable undernotification of vaccination doses was adjusted for in a sensitivity analysis. For the relatively small proportion of electively vaccinated 30-34-year-old women, participation was alarmingly lower than that for their unvaccinated peers.

Concern that vaccinated women may not participate in screening was raised before and during implementation of the National HPV Vaccination Program. A survey of 298 general practitioners undertaken during the program found that $25 \%$ were concerned that HPV vaccine would reduce the uptake of cervical screening. ${ }^{7}$ Program materials targeting young women and parents all included the phrase "The vaccine doesn't prevent all cervical cancers, so regular Pap smears are still essential". This message was reinforced by education and communication materials from the National Cervical Screening Program, which were updated to incorporate the advice that vaccinated women need to continue to participate in screening. ${ }^{8}$ The message focused on the fact that the vaccine only protects against the two HPV types which cause $80 \%$ of cervical cancers; it had less emphasis on the fact that women already sexually active before vaccination may have already acquired HPV infection. Recent analyses from the same linked dataset show that vaccine effectiveness is lower in women who were older at the time of vaccination, reflecting that many of these women were already sexually active at time of vaccination. ${ }^{9}$

Several surveys have provided reassurance that the need for screening despite vaccination was understood by young women. A 2009 populationbased survey found that $96 \%$ of 222 Victorian women aged 18-28 years knew that Pap tests were still needed after vaccination. ${ }^{10}$ In a 2011 population-based mobile phone survey, only 5\% of 1272 young vaccinated women said that they were less likely to have a Pap test because they were vaccinated (own unpublished data). However, our study suggests that this knowledge has not translated into action. This is despite the opportunistic screening during vaccination that was apparent from our data.

The main strength of our study is that we used population-based data, which minimised sampling bias in assessing the relationship between vaccination and screening. Although $\mathrm{HPV}$ vaccinations given to women in the community catch-up program were undernotified to the register, ${ }^{6}$ such underreporting is likely to be independent of whether or not the women were screened and likely to bias the results towards finding no relationship between vaccination status and screening.

The most important limitation of our study is that neither complete identifying data nor Individual Healthcare Identifiers ${ }^{11}$ were available to facilitate linkage of register records. Many young women will change address and Medicare number over a 3-year period, for study or employment reasons, ${ }^{12}$ and $5 \%$ of Victorian women aged 25-29 years marry each year, ${ }^{13}$ potentially resulting in name change. Fifteen of our 16 linkage keys used components of the surname, 10 used all or some of the postcode and 14 used components of the Medicare number. Thus, if notifications for a woman to the two registers used in our study varied by one or more of these variables, the records may not have been linked. This would tend to result in screened women being incorrectly assigned as unvaccinated and vaccinated women being incorrectly assigned as unscreened. Some overmatching could also occur but, on balance, we probably overestimated the negative effect of vaccination on screening participation. However, we do not believe that undermatching can completely explain our findings, which are substantial, consistent over various measures and have public health significance. Our estimates by vaccination status lay either side of and close to the overall published participation rates for screening in each age group. ${ }^{3}$ When we undertook a further assessment of participation, extending the matches used in the analysis to those of lower quality (to try to avoid undermatching, even though this might increase numbers of false matches), we found that our findings were robust for the 25-29-year-olds 
but not for the 20-24-year-olds. It is therefore important that trends in other jurisdictions be explored. We strongly recommend that legislation enabling the operation of cervical screening registers in all Australian jurisdictions be updated to allow disclosure of detailed demographic data to secure linkage units in order to enable linkage of population datasets for public health purposes. Ideally, a process of updating registers such as the VCCR and NHVPR to include Individual Healthcare Identifiers should be prioritised. This would enable more accurate monitoring of the direct effects of vaccination and the effects of vaccination on participation in screening. Another limitation of our study is that the results are not adjusted for socioeconomic status. However, as vaccination and screening are both positively associated with higher socioeconomic status,, 14 socioeconomic differences could not explain the lower participation among vaccinated women.

The National Cervical Screening Program is presently undergoing a renewal process to determine the best screening option for women, given newly available technologies and the impact of the HPV vaccination program. ${ }^{15}$ Raising the starting age for screening to 25 years, in line with guidelines for unvaccinated populations from the International Agency for Research on Cancer and World Health Organization, along with replacing the Pap test with a primary screening test for HPV at 5-year intervals, has been recommended. ${ }^{15}$ The Medical Services Advisory Committee (MSAC) recommended these changes in April 2014. They have not yet been endorsed by the Australian Health Ministers' Advisory Council (AHMAC), but it is anticipated that they will be later this year. The MSAC deliberations and AHMAC considerations constitute the first phase of the renewal. The second phase will be concerned with implementation.

Primary screening with HPV DNA testing will apply to all women, regardless of vaccination status, because it determines whether they are currently infected with a high-risk HPV type. Our data suggest that motivating all young women, whether vaccinated or not, to participate in screening will be critical if the starting age is raised; direct invitations to commence screening, as used for cervical cancer screening in the United Kingdom and breast and bowel cancer screening in Australia, will probably become imperative. ${ }^{16}$

The National Cervical Screening Program renewal process offers an opportunity to reinforce the need for screening among young vaccinated women. In the meantime, efforts to increase participation in screening by vaccinated women are needed.

Acknowledgements: We thank Genevieve Chappell, Michael Malloy, Cathryn May, Karen Winch and the information technology team at the Victorian Cytology Service (VCS) for assisting with data extraction and testing, and staff at the Data Linkage Unit at the AlHW for conducting the data linkage. The VCCR is fully funded by the Victorian Department of Health and operated by the VCS. The NHVPR is owned by the Australian Government Department of Health and operated by the VCS. This study was funded by the VCS. We had full access to all de-identified VCCR and NHVPR data.

Competing interests: Julia Brotherton, Dorota Gertig and Marion Saville were investigators on an Australian Research Council linkage grant on which bioCSL was a partner organisation. Julia Brotherton has been an investigator on investigator-initiated epidemiological research studies which have received partial and unrestricted grants from bioCSL and Merck but has received no personal financial benefits from these grants.

\section{Received 07 Jan 2014, accepted 15 Apr 2014.}

1 Australian Institute of Health and Welfare. Cervical screening in Australia 2010-2011. Canberra: AlHW, 2013. (AlHW Cat. No. CAN 72; Cancer Series No. 76.) http://www. aihw.gov.au/WorkArea/DownloadAsset. aspx?id=60129543399 (accessed Jan 2014).

2 Brotherton JM. How much cervical cancer in Australia is vaccine preventable? A metaanalysis. Vaccine 2008; 26: 250-256.

3 Victorian Cervical Cytology Registry. Statistical report 2012. http://www.vccr.org/downloads/ stat_report_2012.pdf (accessed Feb 2014).
4 Gertig DM, Brotherton JM, Saville M. Measuring human papillomavirus (HPV) vaccination coverage and the role of the National HPV Vaccination Program Register, Australia. Sex Health 2011; 8: 171-178.

5 Brotherton J, Gertig D, Chappell G, et al. Catching up with the catch-up: HPV vaccination coverage data for Australian women aged 18-26 years from the National HPV Vaccination Program Register. Commun Dis Intell Q Rep 2011; 35: 197-201.

6 Brotherton JM, Liu B, Donovan B, et al. Human papillomavirus (HPV) vaccination coverage in young Australian women is higher than previously estimated: independent estimates from a nationally representative mobile phone survey. Vaccine 2014; 32: 592-597.

7 Brotherton JM, Leask J, Jackson C, et al. National survey of general practitioners' experience of delivering the National Human Papillomavirus Vaccination Program. Sex Health 2010; 7: 291-298.

8 Mullins R, Coomber K, Broun K, Wakefield M. Promoting cervical screening after introduction of the human papillomavirus vaccine: the effect of repeated mass media campaigns. J Med Screen 2013; 20: 27-32.

9 Gertig DM, Brotherton JM, Budd AC, et al. Impact of a population-based HPV vaccination program on cervical abnormalities: a data linkage study. BMC Med 2013; 11: 227.

10 Brotherton JM, Mullins RM. Will vaccinated women attend cervical screening? A population based survey of human papillomavirus vaccination and cervical screening among young women in Victoria, Australia. Cancer Epidemiol 2012; 36: 298-302.

11 National E-Health Transition Authority. Healthcare identifiers (HI). http://www.nehta. gov.au/our-work/healthcare-identifiers-hi (accessed Jan 2014).

12 Department of Transport, Planning and Local Infrastructure, State Government of Victoria. Internal migration in Victoria. http://www.dpcd. vic.gov.au/home/publications-and-research/ urban-and-regional-research/census-2011/ internal-migration-in-victoria (accessed Aug 2014).

13 Australian Bureau of Statistics. Marriages and divorces, Australia, 2011. (ABS Cat. No. 3310.0.) http://www.abs.gov.au/AUSSTATS/abs@.nsf/ Lookup/3310.0Main+Features12011?OpenDocu ment (accessed Jan 2014).

14 Barbaro B, Brotherton JML, Gertig DM. Human papillomavirus vaccination and cervical cancer screening by socioeconomic status, Victoria [letter]. Med J Aust 2012; 196: 445.

15 National Cervical Screening Program. National Cervical Screening Program Renewal. http:// www.cancerscreening.gov.au/internet/ screening/publishing.nsf/Content/ncsprenewal (accessed Aug 2014).

16 Canfell K, Sitas F, Beral V. Cervical cancer in Australia and the United Kingdom: comparison of screening policy and uptake, and cancer incidence and mortality. Med J Aust 2006; 185: 482-486. 